\title{
THE TWO-SQUARE LEMMA
}

\author{
Temple H. Fay, Keith A. Harde, and Peter J. Hilton
}

Abstract

A new proof is given of the connecting homomorphism.

One of the most useful, and hence important, lemmas of homological algebra in an abelian category (say, the category of $R$-modules) is the Snake Lemma (see the references at the end of this note). This result is used to produce connecting homomorphisms, building long exact sequences such as the familiar ones involving the Hom - Ext and $\otimes$ - Tor functors. According to Mac Lane [M1], early proofs of this lemma were obscure. One technique, used by Popescu $[\mathrm{P}]$, is to prove the result for abelian groups and use the Mitchell Embedding Theorem to obtain the result in other abelian categories. The essential point is the construction of the homomorphism to do the connecting, not the exactness which comes from simple diagram chasing. In this note we give an interesting new result, which we call the Two-Squere Lemma, that provides a simple and completely categorical construction of the snake connecting morphism. This has the pleasant property of avoiding all references to zig-zags or switchback formulas and to such powerful tools as Mitchell's Embedding Theorem.

We shall do all our work in an abelian category, although some relaxing of the axioms might be possible, say to an exact category. We begin by stating two lemmas which are well-known and fundamental. Indeed apart from these two lemmas we require only the notions of exactness, pullback and pushout in a balanced category.

We record that Beyl $[\mathrm{B}]$ has also given a purely categorical description of the connecting homomorphism. He bases his work on the Product Lemma which is our Lemma 4, and does not enunciate a Two-Square Lemma.

Lemma 1. Consider the commutative diagram

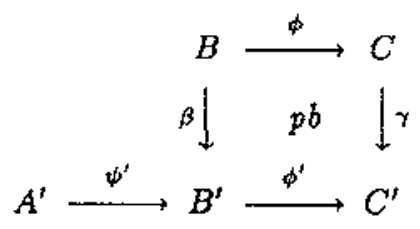


with the bottom row exact. Then there exists a unique morphism $\psi: A^{\prime} \rightarrow B$ such that

$$
\begin{gathered}
\text { (i) } \beta \psi=\psi^{\prime}, \\
\text { and (ii) } A^{\prime} \stackrel{\psi}{\longrightarrow} B \stackrel{\phi}{\longrightarrow} C \text { is exact. }
\end{gathered}
$$

The dual of this result is, of course, also valid.

Lemma 2. (The Sharp $3 \times 3$ Lemma). Consider the following commutative diagram having exact columns and rows.

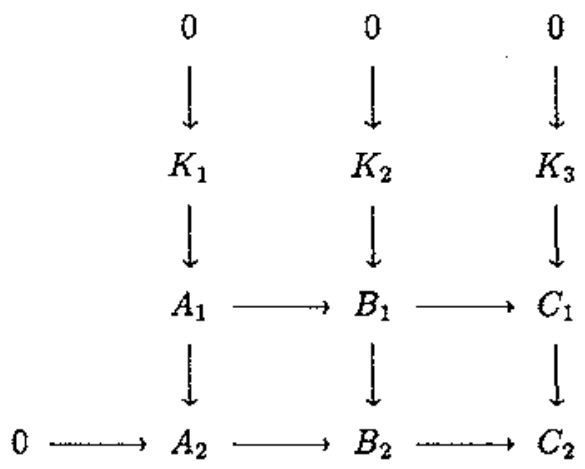

There are uniquely determined morphisms $K_{1} \rightarrow K_{2}$ and $K_{2} \rightarrow K_{3}$ such that the completed diagram is commutative and the sequence

$$
K_{1} \longrightarrow K_{2} \longrightarrow K_{3}
$$

is exact.

Again, the dual of this result is valid. Lemma 2 and its dual provide the two halves of the long exact sequence which the Snake Lemma connects up. The next lemma is the one we wish to emphasize.

Lemma 3. (The Two-Square Lemma) Let the following commutative diagram have exact rows. Form the pull-back of $\gamma$ and $\phi^{\prime}$, and the push-out of $\psi$ and $\alpha$, thus

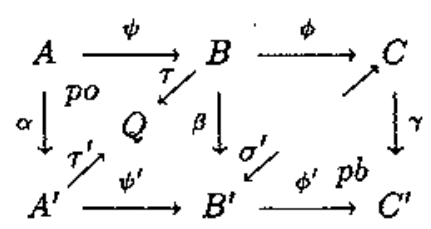




\section{Then}

(i) there exists a unique $\theta: Q \rightarrow B^{\prime}$ such that $\theta r=\beta, \theta \tau^{\prime}=\psi^{\prime}$;

(ii) there exists a unique $\rho: B \rightarrow P$ such that $\sigma \rho=\phi, \sigma^{\prime} \rho=\beta$;

(iii) there exists a unique $\eta: Q \rightarrow P$ such that $\eta \tau=\rho, \sigma^{\prime} \eta=\theta, \sigma \eta \tau^{\prime}=0$. Moreover, $\eta$ is monic if $\psi^{\prime}$ is monic, and $\eta$ is epic if $\phi$ is epic.

Proof: Assertions (i) and (ii) are obvious. To prove (iii), we first use Lemma 1 to find a unique $\mu: A^{\prime} \rightarrow P$ such that $\sigma^{\prime} \mu=\psi^{\prime}$ and $A^{\prime} \stackrel{\mu}{\rightarrow} P \stackrel{\sigma}{\rightarrow} C$ is exact. We then claim that $\mu \alpha=\rho \psi$.

For $\sigma \mu \alpha=0=\phi \psi=\sigma \rho \psi$, and $\sigma^{\prime} \mu \alpha=\psi^{\prime} \alpha=\beta \psi=\sigma^{\prime} \rho \psi$.

Thus there exists a unique $\eta: Q \rightarrow P$ such that $\eta \tau=\rho, \eta \tau^{\prime}=\mu$.

But then $\sigma \eta \tau^{\prime}=\sigma \mu=0$, and $\sigma^{\prime} \eta \tau=\sigma^{\prime} \rho=\beta=\theta \tau, \sigma^{\prime} \eta \tau^{\prime}=\sigma^{\prime} \mu=\psi^{\prime}=\theta \tau^{\prime}$, so that $\sigma^{\prime} \eta=\theta$.

Conversely, if $\eta: Q \rightarrow P$ satisfies $\eta \tau=\rho, \sigma^{\prime} \eta=\theta, \sigma \eta \tau^{\prime}=0$, then $\sigma \eta \tau^{\prime}=0=$ $\sigma \mu, \sigma^{\prime} \eta \tau^{\prime}=\theta \tau^{\prime}=\psi^{\prime}=\sigma^{\prime} \mu$, so that $\eta \tau^{\prime}=\mu$, and the uniqueness of $\eta$ satisfying (iii) is established.

Consider now the commutative diagram

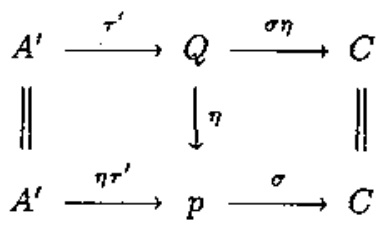

Here the exactress of the top row is obtained by dualizing the argument just given, noting that the definition of $\eta$ is evidently self-dual.

If $\psi^{t}$ is monic, so is $\mu=\eta \tau^{\prime}$. Hence, by the above diagram, so is $\eta$. Dually, if $\phi$ is epic, so is $\eta$.

The final ingredient for the Snake Lemma is the following Triangle Lemma.

Lemma 4. (Triangle Lemma) Given the triangle

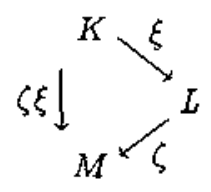

there is an exact sequence

$$
\operatorname{ker} \zeta \xi \longrightarrow \operatorname{ker} \zeta \stackrel{\omega}{\longrightarrow} \operatorname{coker} \xi \longrightarrow \operatorname{coker} \zeta \xi .
$$

Here all morphisms, except possibly $\omega$, are obvious and $\omega$ is the composite

$$
\operatorname{ker} \zeta \longrightarrow L \longrightarrow \operatorname{coker} \xi
$$


of the embedding and the projection.

Proof: Exactness at ker $\zeta$ follows from Lemma 2 applied to the diagram

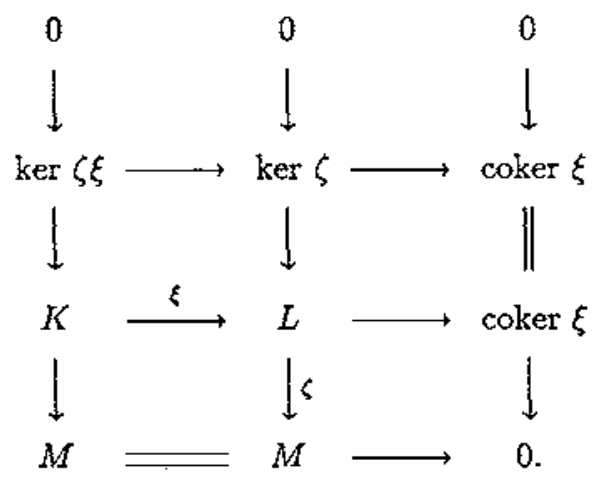

By duality we have also exactness at coker $\xi$.

We are now ready to show how the Snake Lemma follows easily from the Two-Square Lemma.

Lemma 5. (The Snake Lemma) Given the commutative diagram with exact rows

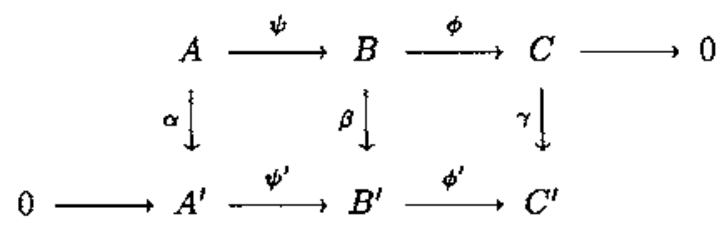

there is a connecting morphism $\omega:$ ker $\gamma \rightarrow$ coker $\alpha$ such that the sequence

$$
\text { ker } \alpha \rightarrow \operatorname{ker} \beta \rightarrow \operatorname{ker} \gamma \stackrel{\omega}{\longrightarrow} \text { coker } \alpha \rightarrow \text { coker } \beta \rightarrow \text { coker } \gamma
$$

is exact.

Proof: The exactriess of ker $\alpha \rightarrow \operatorname{ker} \beta \rightarrow \operatorname{ker} \gamma$ and coker $\alpha \rightarrow \operatorname{coker} \beta \rightarrow$ coker $\gamma$ are just Lemma 2 and its dual. Adopting the notation of the TwoSquare Lemma, we first apply Lemma 1 to obtain a (monic) $\kappa:$ ker $\gamma \rightarrow P$ such that $\sigma \kappa$ is the embedding ker $\gamma \rightarrow C$ and $\operatorname{ker} \gamma \stackrel{\kappa}{\longrightarrow} P \stackrel{\sigma^{\prime}}{\longrightarrow} B^{\prime}$ is exact. Dually we find an epic $\lambda: Q \rightarrow$ coker $\alpha$ such that $\lambda \tau^{\prime}$ is the projection $A^{\prime} \rightarrow \operatorname{coker} \alpha$ and $B \stackrel{\tau}{\longrightarrow} Q \stackrel{\lambda}{\longrightarrow}$ coker $\alpha$ is exact. Exploiting the isomorphism $\eta: Q \rightarrow P$ and the commutative triangle

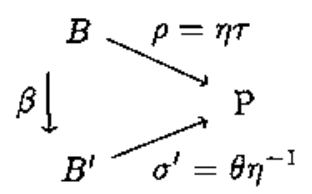


we have $\operatorname{coker} \rho=\operatorname{coker} \tau=\operatorname{coker} \alpha$, $\operatorname{ker} \sigma^{\prime}=\operatorname{ker} \gamma$, so that Lemma 4 yields the exactness of

$$
\operatorname{ker} \beta \rightarrow \operatorname{ker} \gamma \stackrel{\omega}{\longrightarrow} \operatorname{coker} \alpha \rightarrow \operatorname{coker} \beta,
$$

and the proof is complete.

Acknowledgements. The second author acknowledges a grant to the Topology Research Group from the South African Council for Scientific and Industrial Research.

\section{References}

[B] F.R. BEYL, The connecting morphism in the Kernel-Cokernel sequence, Archiv de Math. 32 (1979), 305-308.

[HS] P.J. Hilton aNd U. STAMMBach, "A Course in Homological Algebra," Springer, New York, Heidelberg, Berlin, 1970.

[M1] S. MAC LANE, "Categories for the Working Mathematician," Springer, New York, Heidelberg, Berlin, 1971.

[M2] S. MAC LANE, "Homology," Springer, New York, Heidelberg, Berlin, 1963.

[M] B. Mitchell, Theory of Categories, Academic Press (1965), London, New York.

[P] N. Popescu, Abelian Categories with Applications to Rings and Modules, Academic Press (1973), London, New York.

[H] H. SChUBERT, "Categories," Springer, New York, Heidelberg, Berlin, 1972 .

Temple H. Fay: Department of Mathematics

Univ. of Southern Mississippi

Hattiesburg, MS 39406

U S A

Peter J. Hilton: Department of Math. Sciences

State Univ. of New York

Binghamton, NY 13901

U S A

Keith A. Hardie: Department of Mathematics

Univ, of Cape Towm

Rondebosch 7700

SOUTH AFRICA

Rebut el 16 de juny de 1988 